\section{A Local Approximation of the Cable Equation for Implementing a Local Interaction Model}

\author{
Jaap Hoekstra \\ Delft University of Technology, \\ Dept. Electrical Engineering, \\ P.O. Box 5031, 2600GA Delft, The Netherlands \\ e-mail: jaap@neuron.et.tudelft.nl
}

This paper discusses a reverse engineering approach to artificial neural networks. The starting-point chosen for the reverse engineering process is the local interaction model. The engineering step models this local interaction model by an electronic ladder network, in which an input signal causes a potential pulse that rapidly decreases in amplitude during transport toward the end of the network. The mathematical model of this step consists of a system of coupled differential equations that solve the "cable equation". The computational model of this step consists of a local approximation of the cable equation, such that local leaming can be computed by local computations. As a consequence of the model learning is local and dependent on the place where input signals enter the dendrite and on the temporal relation of these inputs.

The starting-point chosen for the reverse engineering process is the local interaction model described by Alkon [1]. local interaction between post-synaptic sites is assumed. I have taken some of Alkon's design principles as a basis for an electrical model, describing local interactions, that is simulated by a local approximation of the dendritic cable equation.

Models incorporating local leaming rules allow increase and decrease of weights based on activities of connected nodes if the synapses are placed close together on the same dendrite. These models model the biological dendrite by solving an equivalent electrical circuit, consisting of several compartments. Current models solve the electrical circuit model by the numerical calculation of a recurrence relation in which the current of a compartment is expressed in the value of the current in a next compartment, see for example [2]. In this way it is always necessary to solve the whole dendrite, even if we are only interested in local effects. In this paper an altemative mathematical description is used on bases of which local activities can be calculated by only simulating a part of the dendrite model.

The engineering step models this local interaction model by an electronic ladder network (for the dendritic cable) in which an input signal causes a potential pulse, $V_{k}(t)$, that rapidly decreases in amplitude during transport toward the end of the network (representing the cell body). The leaming rule can now be formulated by:

$\Delta \omega_{i j}(t) \sim \eta V_{k}(t)$

where $\omega_{i j}$ is the weight between the input from node (neuron) $j$ at the place $k$ in the network, $\eta$ is a leaming rate.

Basic to a local leaming scheme is that a voltage pulse induced at a specific compartment only affects other compartments within a limited range. In relation to the above described electrical model it means that the influence of a voltage pulse strongly decreases after a limited number of compartments. And on the other hand that the effect of neighboring compartments on a voltage pulse is only limited to a small number of them.

The compartmental voltage can be approached by

For $: h \leq h_{\max }$ :

$V_{k}=V_{k-h}-\sum_{j=k-h+1}^{k} r_{f-1, f} \sum_{l=f}^{k_{\max }}\left(c_{m_{l}} \frac{d V_{l}}{d t}+\frac{V_{l}}{r_{m_{l}}}-I_{j_{l}}\right)$

Simulations show that for values of the capacitance and resistors not far besides biological plausible values a value of 5 for both $k_{\max }$ and $h_{\max }$ can be obtained.

The description above has an interesting feature. Recall that the model input was a one dimensional dendrite in which the transport direction of the charge was only toward the cell body, thus in one direction. There is, however, an influence of a limited number of capacitances from the potential in compartments between the compartment and the cell body. The height of the potential not only depends on previous (further away from the cell body) compartments, but also on how many following capacitances have to be charged. This last number also depends on stimuli present on following compartments. Thus, even in this model, the potential is determined by stimuli from two directions.

\section{References}

[1] D.L. Alkon, 'Memory Storage and Neural Systems', In: Scientific American, July 1989.

[2] Methods in Neuronal Modeling, C. Koch and I. Segev (Eds.), Cambridge MA: MIT Press, pp 63-97, 1989. 